\title{
Spectral variability modes of GX 339-4 in a hard-to-soft state transition
}

\author{
Melania Del Santo* \\ INAF/IASF-Roma, Italy \\ E-mail: melania.delsanto@iasf-roma.inaf.it \\ Julien Malzac \\ CNRS/CESR Toulouse, France
}

\section{Pietro Ubertini}

INAF/IASF-Roma, Italy

\section{Tomaso Belloni}

INAF/OAB Merate, Italy

\begin{abstract}
We report on INTEGRAL observations performed during the 2004 outburst of the bright black hole transient GX 339-4. We analysed IBIS and JEM-X public data starting on 9th August and lasting about one month. During this period GX 339-4 showed spectral state transitions. In order to seek for variability patterns, a principal component analysis (PCA) has been used.
\end{abstract}

VI Microquasar Workshop: Microquasars and Beyond

September 18-22 2006

Società del Casino, Como, Italy

${ }^{*}$ Speaker. 

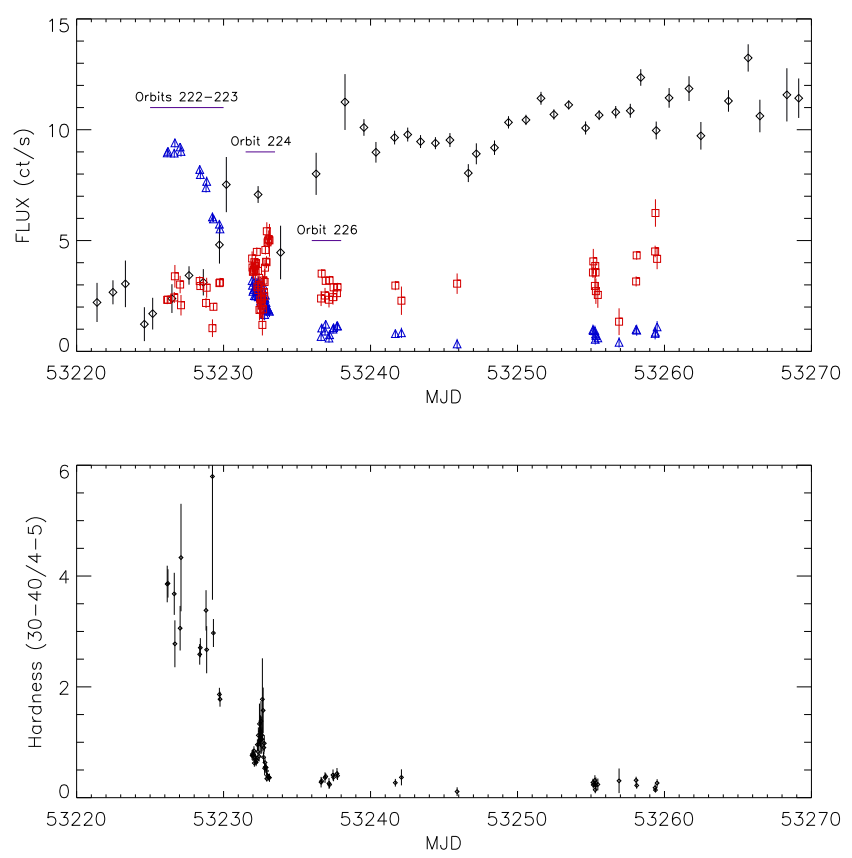

Figure 1: GX 339-4 count rate in $30-40 \mathrm{keV}, 4-5 \mathrm{keV}$ and $1.5-3 \mathrm{keV}$ with IBIS/ISGRI (blue triangles), JEM-X (red squares) and ASM (black diamonds), respectively (top). IBIS/ISGRI to JEM-X hardness ratio (bottom).

\section{Introduction}

Classified as black-hole candidate [13], GX 339-4 is a transient source spending long periods in outburst. Before the launch of RXTE the source has been prevalently observed in the Low/Hard (LH) spectral state, even though several transitions to softer states were reported [2]. Thereafter GX 339-4 remained bright and mostly in the LH state until 1999 when it went into quiescence. After the quiescent state which was observed by BeppoSAX [8], [6], GX 339-4 showed two new outbursts: in 2002/2003 [12], [11], [2] and in 2004 after one year in quiescence [1], [5]. The longterm variability of GX 339-4 and a theoretical interpretation referred to the time range 1987-2004 (only the first LH period for the latter) is extensively described in Zdziarski et al. (2004). These authors studied in detail the hysteresis-like behaviour, especially the strong dependence of the flux of the hard-to-soft transition on the preceding behaviour of the hard/quiescent state. The 2004 outburst started on February and occurred at luminosity levels lower than the previous one. Joint RXTE/INTEGRAL observations performed on 2004 August $14^{\text {th }}-16^{\text {th }}$ are presented in Belloni et al. (2006). These authors report on the first determination for a BHC of the changes of the broad-band X-ray spectrum across the High Intermediate State (HIMS)-Soft Intermediate State (SIMS) fast transition [3].

In order to seek for a variability pattern of Cyg X-1, Malzac et al. (2006) used a Principal Component Analysis (PCA) during an intermediate state of that source. These authors found that the spectral variability occurred through two dependent modes: the first consisted in changes in the overall luminosity on time scale of hours with almost constant spectrum (responsible for $68 \%$ of 

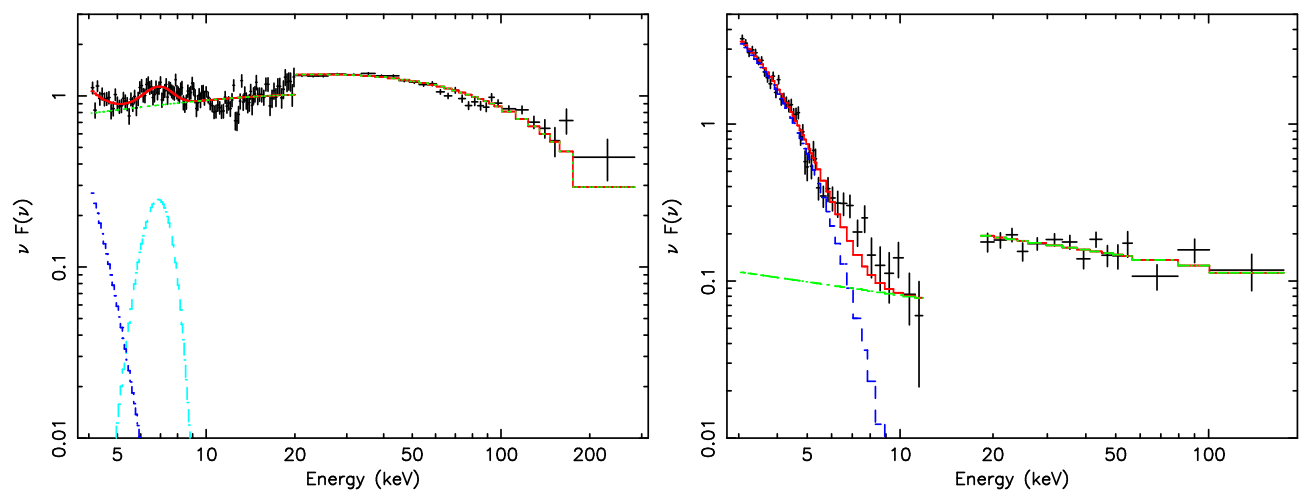

Figure 2: Energy spectrum (erg cm ${ }^{-2} \mathrm{~s}^{-1}$ ), total model and single components of the HIMS (left) and SIMS (right) spectra. The JEM-X and IBIS/ISGRI spectra shown are not normalised.

the variance); the second mode consisted in a pivoting of the spectrum around $10 \mathrm{keV}$ (27\% of the variance).

In this work we present GX 339-4 spectral analysis of INTEGRAL data collected in the two adjacent periods to the one used by Belloni et al. (2006), as well as a PCA on the spectral transition period.

\section{Observations and data analysis}

We used INTEGRAL data collected during the period 9 August-10 September 2004, which covered range $222 \div 233$ of the satellite orbits. In particular, we selected a data sub-set with all pointings common to JEM-X and IBIS telescopes for a total of 76 Science Windows (SCWs), lasting 1800-3600 seconds each. INTEGRAL data analysis has been performed with OSA v. 5.1.

Light curves for each pointing were extracted in 15 bins ${ }^{1}$. The IBIS/ISGRI and the JEM-X temporal behaviours are shown in Fig. 1. The Belloni et al. (2006) data correspond to orbit 224, during which the transition occurred.

Spectral fitting of two average spectra (specl and spec2), collected within orbits 222-223 and 226, respectively, is presented. We used simple models as multicolor disc and cut-off or simple power laws in XSPEC v. 11.3.1. The single parameters uncertainties have been calculated at the $90 \%$ confidence level and the cross-calibration constant is 0.7 with 0.2 of uncertainty (const $=1$ for IBIS/ISGRI).

\subsection{The principal Component Analysis}

Principal Component Analysis (PCA) is a powerful tool for multivariate data analysis used for a broad range of applications in natural as well as social science [7]. The main use of PCA is to reduce the dimensionality of a data set while keeping as much informations as possible. PCA transforms a number of (possibly) correlated variable in a smaller number of uncorrelated variables called principal components.

Details on the used method are described in Malzac et al. (2006). In our case, $p=76$ spectra (one for each SCW) binned into $n=15$ bins corresponding to energies $E_{1}, E_{2}, \ldots, E_{n}$.

\footnotetext{
${ }^{1}$ Namely: 3-4, 4-5, 5-6, 6-7, 7-9, 9-11, 11-13, 13-15, 15-20, 20-30, 30-40, 40-50, 50-70, 70-100, 100-200 keV
} 

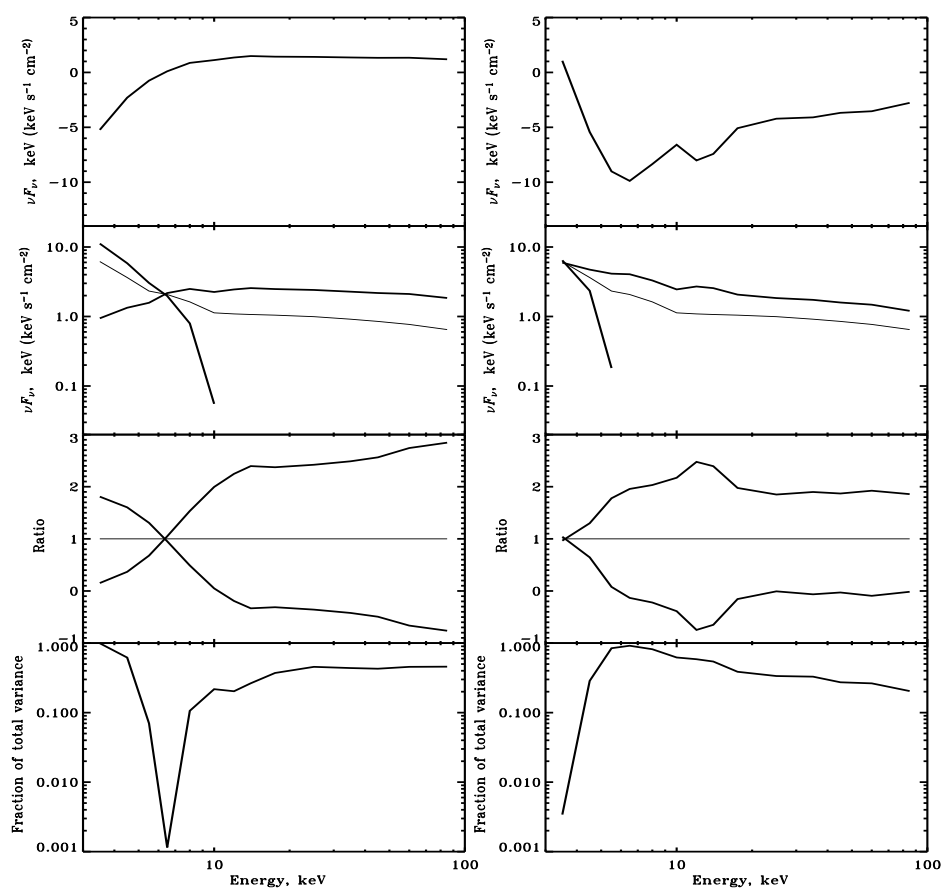

Figure 3: PC1 (left) and PC2 (right). From the top panel: the shape of the principal component, the effect on the shape and normalisation of the spectrum, the ratios maximum (and minimum) spectrum to the average one and the contribution to the variance are shown.

\section{Results}

The hardness ratio $30-40 \mathrm{keV}$ to $4-5 \mathrm{keV}$ (Fig. 1, bottom) clearly shows the progressive softening of GX 339-4; the spectral transition to the SIMS state occurred between MJD 53232 and MJD 53233 [4]. In the HIMS the best-fit parameters have been found to be $\Gamma=1.72 \pm 0.04, \mathrm{E}_{c}=89 \pm 3$ $\mathrm{keV}$ and $\mathrm{kT}_{\text {diskbb }}=0.36 \pm 0.01 \mathrm{keV}$ (Fig. 2, left). The Fe line has been frozen at $6.7 \mathrm{keV}$ and may be an instrumental effect. In Fig. 2 (right) the SIMS energy spectrum (spec2) and the best-fit model being $\Gamma=2.2 \pm 0.01$ and $\mathrm{kT}_{\text {diskbb }}=0.61 \pm 0.02 \mathrm{keV}$ are shown.

Two principal components describing the GX 339-4 variability have been found: PC1 responsible for $75 \%$ of the variance and PC2 for $21 \%$. In Fig. 3 we present from the top to the bottom:

1 the shape of each PC which would be added or subtracted to the average spectrum in order to reproduce the variability;

2 the effects of PC1 and PC2 on the shape and normalisation of the spectrum: time-averaged spectrum (light line) and spectra obtained for the maximum and minimum (solid lines) observed values of the normalisation parameter;

3 the ratio of the maximum and minimum spectra to the average one;

4 the contribution of each component to the total variance as a function energy. 


\section{Discussion}

We have presented INTEGRAL observations of GX 339-4, which covered part of the 2004 outburst. We followed the source spectral evolution through the Hard-Intermediate and SoftIntermediate states, until a softer state. The variability of GX 339-4 can be described by two independent modes:

- spectral evolution with the spectrum pivoting around $6 \mathrm{keV}(\mathrm{PC} 1)$ contributing at $75 \%$. This pivoting mode may be interpreted as caused by changes in the soft cooling photons flux in the hot Comptonising plasma associated with an increase of temperature of the accretion disc.

- intensity variation of the hard power-law component (almost constant slope) on top of constant soft component (PC2) contributing at $21 \%$. It would be interpreted to be due to magnetic flares occurring in the corona.

Unlike Cyg X-1 [9], the pivoting mode has been found to be more important in GX 339-4. Reasonably, because Cyg X-1 was observed during an incomplete spectral transition hard-tointermediate, while GX 339-4 achieved finally a softer state.

\section{Acknowledgements}

This work has been supported by the Italian Space Agency grant I/R/046/04. Based on observations with INTEGRAL, an ESA project with instruments and science data centre funded by ESA member states (especially the PI countries: Denmark, France, Germany, Italy, Switzerland, Spain), Czech Republic and Poland, and with participation of Russia and the USA. MDS thanks Angela Bazzano for precious suggestions and support.

\section{References}

[1] Belloni, T., et al. 2004, ATel \#236

[2] Belloni, T., et al. 2005a, A\&A, 440, 207

[3] Belloni, T., 2005b, Proceedings of COSPAR Colloquium "Spectra \& Timing of Compact X-ray Binaries," 2005, Mumbai, India

[4] Belloni, T., et al. 2006, MNRAS, 367, 1113

[5] Buxton, M., et al. 2004, ATel \#230

[6] Corbel, S., et al. 2003, A\&A, 400, 1007

[7] Kendall, M. G. 1980, "Multivariate analysis", ed. Griffin \& co. London

[8] Kong, A. K. H., et al. 2000, MNRAS, 312, L49

[9] Malzac, J., et al. 2006, A\&A, 448, 1125

[10] Miyamoto, S., et al. 1991, ApJ, 435, 398

[11] Nespoli, E., et al. 2003, A\&A, 412, 235

[12] Smith, D. M., et al. 2002, ATel \#95

[13] Zdziarski, A. A., et al. 1998, MNRAS, 301, 435

[14] Zdziarski, A. A., et al. 2004, MNRAS, 351, 791 